\title{
$P$-ADIC TRANSCENDENTAL NUMBERS
}

\author{
KUMIKO NISHIOKA
}

(Communicated by William Adams)

\begin{abstract}
Explicit sets of cardinality $2^{\aleph_{0}}$ of $p$-adic numbers which are algebraically independent over $Q_{p}$ are constructed.
\end{abstract}

Let $\mathbf{Q}_{p}$ be the $p$-adic completion of $\mathbf{Q}$ for a prime $p$. Let $\overline{\mathbf{Q}}_{p}$ be the algebraic closure of $\mathbf{Q}_{p}$, and $\mathbf{C}_{p}$ be its $p$-adic completion which is an algebraically closed field of cardinality $2^{\aleph_{0}}$. Let $\mathbf{Q}_{p}^{\text {unram }}$ be the maximum unramified extension field of $\mathbf{Q}_{p}$. Then $\mathbf{Q}_{p}^{\text {unram }}=\mathbf{Q}_{p}(W)$, where $W$ is the set of all roots of unity whose orders are prime to $p$. Let $\mathbf{C}_{p}^{\text {unram }}$ be the $p$-adic closure of $\mathbf{Q}_{p}^{\text {unram }}$ in $\mathbf{C}_{p}$. Koblitz [1] asked whether $\mathbf{C}_{p}^{\text {unram }}$ has uncountably infinite transcendence degree over $\mathbf{Q}_{p}$ and $\mathbf{C}_{p}$ has uncountably infinite transcendence degree over $\mathbf{C}_{p}^{\text {unram }}$. Lampert [2] answered that the transcendence degree of $\mathbf{C}_{p}^{\mathrm{unram}}$ over $\mathbf{Q}_{p}$ is $2^{\aleph_{0}}$ and the transcendence degree of $\mathbf{C}_{p}$ over $\mathbf{C}_{p}^{\mathrm{unram}}$ is $2^{\aleph_{0}}$ by constructing sets of algebraically independent numbers of cardinality $2^{\aleph_{0}}$. Here we will give more explicit examples of such sets which cannot be obtained by the method in [2].

Theorem. Let $K$ be an intermediate field between $\mathbf{Q}_{p}$ and $\mathbf{C}_{p}$. Let $\alpha_{1}, \ldots, \alpha_{m}$ be in $\mathbf{C}_{p}$ and $\alpha_{1}, \ldots, \alpha_{m-1}$ be algebraically independent over $K$. Suppose that for $i=1, \ldots, m-1$ there exist sequences $\left\{\beta_{i k}\right\}_{k \geq 1}$ in $\mathbf{C}_{p}$ converging to $\alpha_{i}$ and a sequence $\left\{S_{k}\right\}_{k \geq 1}$ of finite subsets of $\operatorname{Aut}\left(\mathbf{C}_{p} / K\left(\left\{\boldsymbol{\beta}_{i k}\right\}_{1 \leq i \leq m-1}\right)\right)$ which satisfies

$$
\begin{aligned}
& \lim _{k \rightarrow \infty}\left|S_{k}\right|=\infty \text { and } \alpha_{m}^{\sigma} \neq \alpha_{m}^{\tau} \text { for any } \sigma, \tau \in S_{k} \text { with } \sigma \neq \tau, \\
& \max _{1 \leq i \leq m-1}\left|\alpha_{i}-\beta_{i k}\right|_{p}=o\left(\min _{\substack{\sigma, \tau \in S_{k} \\
\sigma \neq \tau}}\left|\alpha_{m}^{\sigma}-\alpha_{m}^{\tau}\right|_{p}\right) \quad \text { as } k \rightarrow \infty,
\end{aligned}
$$

where we define the left-hand side of (2) to be 0 if $m=1$. Then $\alpha_{1}, \ldots, \alpha_{m}$ are algebraically independent over $K$.

To prove the theorem we need the following lemma which is proved in Koblitz [1].

Received by the editors March 13, 1989.

1980 Mathematics Subject Classification (1985 Revision). Primary $11 \mathrm{~J} 61$. 
Lemma (Koblitz [1], p. 70). Let $f(X) \in \mathbf{C}_{p}[X]$ have degree $n$,

$$
f(X)=a_{n} X^{n}+a_{n-1} X^{n-1}+\cdots+a_{1} X+a_{0} .
$$

Suppose that $f(X)$ has no multiple root. Then there exists a positive constant $c$ such that if $g(X)=\sum_{i=0}^{n} b_{i} X^{i} \in \mathbf{C}_{p}[X]$ has degree $n$, and if $\max _{0 \leq i \leq n}\left|a_{i}-b_{i}\right|_{p}$ is sufficiently small, then for every root $\beta$ of $g(X)$ there is precisely one root $\alpha$ of $f(X)$ such that

$$
|\alpha-\beta|_{p} \leq \max _{1 \leq i \leq n}\left|a_{i}-b_{i}\right|_{p} .
$$

Proof of theorem. Suppose that $\alpha_{1}, \ldots, \alpha_{m}$ are algebraically dependent over $K$. Then there exists a polynomial $f(X)$ of degree $n$ with coefficients in $K\left[\alpha_{1}, \ldots, \alpha_{m-1}\right]$,

$$
f(X)=Q_{n}\left(\alpha_{1}, \ldots, \alpha_{m-1}\right) X^{n}+\cdots+Q_{0}\left(\alpha_{1}, \ldots, \alpha_{m-1}\right)
$$

such that $f\left(\alpha_{m}\right)=0$ and $f(X)$ has no multiple root. If $\sigma \in S_{k}$, then

$$
\begin{aligned}
& \left|Q_{i}\left(\alpha_{i}^{\sigma}, \ldots, \alpha_{m-1}^{\sigma}\right)-Q_{i}\left(\alpha_{1}, \ldots, \alpha_{m-1}\right)\right|_{p} \\
& \quad \leq \max \left\{\left|Q_{i}\left(\alpha_{1}^{\sigma}, \ldots, \alpha_{m-1}^{\sigma}\right)-Q_{i}\left(\beta_{1 k}, \ldots, \beta_{m-1, k}\right)\right|_{p},\right. \\
& \left.\quad \leq Q_{i}\left(\beta_{1 k}, \ldots, \beta_{m-1, k}\right)-\left.Q_{i}\left(\alpha_{1}, \ldots, \alpha_{m}\right)\right|_{p}\right\} \\
& \quad \leq c_{1} \max _{1 \leq i \leq m-1}\left|\alpha_{i}-\beta_{i k}\right|_{p},
\end{aligned}
$$

where $c_{1}$ is a positive constant. If $k$ is sufficiently large, then $\left|S_{k}\right|>n$ and by the lemma, there exists a root $\alpha$ of $f(X)$ and two distinct elements $\sigma, \tau$ of $S_{k}$ such that

$$
\left|\alpha-\alpha_{m}^{\sigma}\right|_{p},\left|\alpha-\alpha_{m}^{\tau}\right|_{p} \leq c_{2} \max _{1 \leq i \leq m-1}\left|\alpha_{i}-\beta_{i k}\right|_{p}
$$

where $c_{2}$ is a positive constant, and so

$$
\min _{\substack{\sigma, \tau \in S_{k} \\ \sigma \neq \tau}}\left|\alpha_{m}^{\sigma}-\alpha_{m}^{\tau}\right|_{p} \leq c_{2} \max _{1 \leq i \leq m-1}\left|\alpha_{i}-\beta_{i k}\right|_{p}
$$

This contradicts condition (2) and the theorem is proved.

It is well known that every element $\alpha$ of $\mathbf{C}_{p}^{\text {unram }}$ is uniquely represented as $\alpha=\sum_{n \geq q} \zeta_{n} p^{n}$ where $\zeta_{n} \in W$ and $q \in \mathbf{Z}$. The number $\alpha$ is transcendental over $\mathbf{Q}_{p}$ if and only if the extension degree $\left[\mathbf{Q}_{p}\left(\zeta_{n}\right): \mathbf{Q}_{p}\right], n \geq q$, is unbounded. By using the theorem, we obtain a set of cardinality $2^{\aleph_{0}}$ whose elements are in $\mathbf{C}_{p}^{\text {unram }}$ and algebraically independent over $\mathbf{Q}_{p}$.

Example 1. Let $\zeta(n)$ be a primitive $n$th root of unity for every natural number $n$. Let $P$ be the set of all prime numbers. Then the numbers

$$
\sum_{n=1}^{\infty} \zeta\left(l^{[\lambda n]}\right) p^{n}, \quad\left(l \in P-\{p\}, \lambda \in \mathbf{R}^{+}\right)
$$

are algebraically independent over $\mathbf{Q}_{p}$. 
Proof. Let $l_{1}, \ldots, l_{s} \in P-\{p\}$ and $K$ be the $p$-adic closure of $\mathbf{Q}_{p}\left(\left\{\zeta\left(l_{i}^{n}\right)\right\}_{1 \leq i \leq s, n \geq 0}\right)$. Let $l \in P-\left\{p, l_{1}, \ldots, l_{s}\right\}$ and $0<\lambda_{1}<\cdots<\lambda_{m}$. Put

$$
\alpha_{i}=\sum_{n=0}^{\infty} \zeta\left(l^{\left[\lambda_{i} n\right]}\right) p^{n}, \quad 1 \leq i \leq m .
$$

It is enough to prove that $\alpha_{1}, \ldots, \alpha_{m}$ are algebraically independent over $K$. We prove it by induction on $m$. Assume that $\alpha_{1}, \ldots, \alpha_{m-1}$ are algebraically independent over $K$. Put

$$
\boldsymbol{\beta}_{i k}=\sum_{n=1}^{k+[\log k]} \zeta\left(l^{\left[\lambda_{i} n\right]}\right) p^{n}, \quad 1 \leq i \leq m-1, k \geq 1,
$$

and

$$
d_{k}=\left[K\left(\zeta\left(l^{\left[\lambda_{m} k\right]}\right)\right): K\left(\zeta\left(l^{\left[\lambda_{m-1}(k+[\log k])\right]}\right)\right)\right] .
$$

Then

$$
\left|\alpha_{i}-\beta_{i k}\right|_{p}=p^{-k-[\log k]-1}
$$

and $\lim _{k \rightarrow \infty} d_{k}=\infty$. Let $S_{k}$ be a set of $d_{k}$ isomorphisms of $\mathbf{C}_{p}$ which is obtained by extending $\operatorname{Gal}\left(K\left(\zeta\left(l^{\left[\lambda_{m} k\right]}\right)\right) / K\left(\zeta\left(l^{\left[\lambda_{m-1}(k+[\log k])\right]}\right)\right)\right)$. Then

$$
\min _{\substack{\sigma, \tau \in S_{k} \\ \sigma \neq \tau}}\left|\alpha_{m}^{\sigma}-\alpha_{m}^{\tau}\right|_{p} \geq p^{-k}
$$

Hence by the theorem, $\alpha_{1}, \ldots, \alpha_{m}$ are algebraically independent over $K$.

In a similar way, we obtain a set of cardinality $2^{\aleph_{0}}$ whose elements are in $\mathbf{C}_{p}$ and algebraically independent over $\mathbf{C}_{p}^{\mathrm{unram}}$.

Example 2. The numbers

$$
\sum_{n=1}^{\infty} p^{n+l^{-[i, n]}}, \quad\left(l \in P-\{p\}, \lambda \in \mathbf{R}^{+}\right)
$$

are algebraically independent over $\mathbf{C}_{p}^{\mathrm{unram}}$.

\section{REFERENCES}

1. N. Koblitz, P-adic number theory, p-adic analysis and zeta functions, G. T. M. Vol. 58, Springer-Verlag, New York, 1977.

2. D. Lampert, Algehraic P-adic expansions, J. Number Theory 23 (1986), 279-284.

Nara Women's University, Department of Mathematics, Kita-Uoya Nishimachi, Nara 630, JAPAN 